\title{
Hospital Infection Prevention: How Much Can We Prevent and How Hard Should We Try?
}

\author{
Gonzalo Bearman $^{1} \cdot$ Michelle Doll $^{1} \cdot$ Kaila Cooper $^{1} \cdot$ Michael P. Stevens $^{1}$
}

Published online: 2 February 2019

(C) Springer Science+Business Media, LLC, part of Springer Nature 2019

\begin{abstract}
Purpose of Review To summarize the extent to which hospital-acquired infections (HAIs) are preventable and to assess expectations, challenges, and barriers to improve patient outcomes.

Recent Findings HAIs cause significant morbidity and mortality. Getting to zero HAIs is a commonly stated goal yet leads to unrealistic expectations. The extent to which all HAIs can be prevented remains debatable and is subject to multiple considerations and barriers. Current infection prevention science is inexact and evolving. Evidence-based infection prevention practices are often incompletely implemented and at times controversial. Highly sensitive surveillance results in overdiagnosis, calling into question the real incidence of HAIs. Perceived reductions in HAIs by gaming the system lead to false conclusions about preventability and may cause harm. Successful HAI reduction programs require executive oversight yet keeping hospital leaders engaged in infection prevention is a challenge given competing priorities. Medicine is not a physical science with precisely defined laws; thus, infection prevention interventions are subject to variable outcomes.

Summary Perhaps up to 55-70\% of HAIs are potentially preventable. This is subject to a law of diminishing returns as the preventable proportion of HAIs may reduce over time with improvements in patient safety. As the principle tenet of medicine is first do no harm, infection prevention programs should relentlessly pursue reliable, sustainable, and practical strategies for heightened patient safety.
\end{abstract}

Keywords Infection prevention · Patient safety $\cdot$ Implementation science $\cdot$ Hospital epidemiology $\cdot$ Public health $\cdot$ Healthcare quality

\section{Introduction}

Hospital-acquired infections remain a primary focus of healthcare safety and quality programs. Despite advances in the science of infection prevention, the impact of hospitalassociated infections (HAIs) is significant. In 2002, Klevens et al. reported an aggregate of greater than 1.7 million HAIs in North America, with 98,987 deaths and a corresponding fatality rate of $5.7 \%$ [1]. A more recent meta-analysis, published in 2013, estimated the financial impact on the US healthcare

This article is part of the Topical Collection on Healthcare Associated Infections

Gonzalo Bearman

gonzalo.bearman@vcuhealth.org

1 Virginia Commowealth University Hospital Infection Prevention Program, North Hospital, 2nd Floor, Room 2-073, 1300 East Marshall Street, Richmond, VA 23298-0019, USA system. Estimated costs were $>\$ 27$ million for catheter associated urinary tract infections (CAUTI), $>\$ 1$ billion each for central line associated bloodstream infections (CLABSIs) and C. difficile infections, and $>\$ 3$ billion each for surgical site infections (SSIs) and ventilator associated pneumonia (VAP) [2]. By current estimate, $3.2 \%$ of all hospitalized patients have an HAI [ $\left.3 \bullet^{\bullet}\right]$. If that were insufficient, regulatory agencies and public reporting of HAIs obligate us to act and prioritize HAI risk reduction strategies. Doing nothing is not an option.

\section{Zero Hospital-Acquired Infections: Reality Check}

Isolated reports of infection prevention campaigns resulting in zero hospital-acquired infections exist. In the most recent hospital survey by the Leapfrog Group, $12.7 \%$ of the almost 2000 participating acute care hospitals reported zero CLABSIs, $11.3 \%$ zero CAUTIs, $14.6 \%$ zero methicillin resistant 
Staphylococcus aureus (MRSA) HAIs, 2.8\% zero hospital onset $C$. difficile, and $19.2 \%$ zero colon surgery SSIs [4••]. The group notes that the number of hospitals reporting zero infections is falling since 2015, despite the fact that overall hospitals are improving their rates [4••]. This suggests that increased awareness and reporting of HAIs may be responsible for the drop in the number of hospitals claiming zero HAIs over the past year. In the published scientific literature, outcomes of zero events are of limited time frame and in select patient populations [5], raising significant doubt about both reproducibility and sustainability to scale.

For some, the prospect of "getting to zero" HAIs is the Holy Grail of the heightened patient safety quest. In the history of medicine, global disease elimination exists only for smallpox. As previously explored by Edmond, getting to zero HAIs is best described as a sound bite as it lacks complexity and nuance, is misleading, is based on inexact science with suboptimal evidence, and ultimately results in unrealistic expectations and outcomes [6].

\section{Implementation of Infection Prevention Best Practices: We Are Not As Good As We Think}

Recent, seminal publications in infection prevention include works on central line insertion standardization (checklist), the use of chlorhexidine gluconate impregnated dressings (CHG sponge), and patient bathing with an antiseptic. Berenholtz et al. reported significant reductions in CLABSI across surgical intensive care unit populations at a major US academic healthcare center by employing an evidence based central line insertion checklist, completed at the point of care by the bedside nurse overseeing the procedure [7]. The study authors reported $63 \%$ global compliance with checklist completion. The same central line checklist protocol was later adopted across 103 Michigan ICUs, again with significant reductions in CLABSI rates across participating institutions [5]. Global compliance with central line checklist completion is unknown as the investigators "...did not evaluate compliance with study intervention." [5] Timsit et al. performed a randomized, blinded controlled trial conducted in seven French ICUs comparing CHG sponge vs. standard dressings (controls) with an outcome of catheter related bloodstream infections (cr-BSIs) [8]. Significant reductions in cr-BSIs were reported despite unclear fidelity with the use of the CHG sponge dressings. Global compliance with central line dressing use was unclear with " $50 \%$ of dressing changes were unplanned." Last, a landmark multicenter, cluster-randomized, crossover trial of daily bathing with CHG-impregnated washcloths versus standard patient bathing reported statistically significant reductions in acquisition of multidrug resistant organisms (MDROs) and the incidence of hospital-acquired bloodstream infections [9]. Compliance with patient bathing per protocol was not reported. Thus, even in the most seemingly robust of oversight scenarios, such as during investigative trials, infection prevention processes are (at best) variably implemented.

In diverse patient populations, compliance with infection prevention process of care measures is similarly challenged. A Canadian survey of 154 hospitals found variable implementation of interventions to reduce $C$. difficile, with $27 \%$ isolating patients at onset of diarrhea, $16 \%$ performing antibiotic stewardship audits, and $72 \%$ monitoring cleaning practices [10]. Fakhii et al. performed a survey of select key infection prevention practices across 71 US hospitals [11]. The use of urinary catheter electronic reminders to nurses (for catheter awareness and discontinuation) was employed in $14 \%$ of hospitals. Pre-operative glucose monitoring $>90 \%$ of time was reported by $18 \%$ of hospitals; use of central line insertion checklist $>90 \%$ of time, $60 \%$ of respondents; weight-based perioperative antibiotic dosing, $64 \%$ of respondents; and daily evaluation of sedation vacation, for ventilator associated pneumonia risk reduction, reported by $93 \%$ of respondents. When we stand in front of the mirror of infection prevention compliance, we are faced with a less than fulfilling image of infection prevention risk reduction reliability.

\section{Controversy in Infection Prevention: The Contact Precautions for Endemic Pathogens Debate}

Although contact precaution for MRSA and vancomycin resistant enterococcus (VRE) colonized and infected patients is an established infection prevention strategy, the practice is controversial. The greater the burden of contact precaution use, the less the compliance with both hand hygiene and the contact precaution bundle-appropriate use of gown and gloves [12]. The goal is to optimize the burden of contact precautions while sustaining compliance with gowns, gloves, and hand hygiene. Robust measures for the incremental benefit of contact precautions, gowns, gloves, and active detection and isolation strategies for the prevention of MRSA and VRE cross-transmission in endemic settings are lacking [13].

A cluster randomized trial of universal gloving and gowning (universal contact precautions) across 20 medical and surgical ICUs in 20 US hospitals assessed the impact of patient contact isolation on both adverse events and clinically centered infection prevention outcomes [14]. Universal gloving and gowning did not reduce primary VRE or MRSA acquisition yet reduced secondary MRSA acquisition by $40 \%$ relative risk. No difference in adverse events was reported. The benefit was small relative to isolation burden and effort of gown and glove use.

Several medical centers reported no negative impact on MRSA and VRE rates after the discontinuation of contact precautions. Using a quasi-experimental study design, Edmond et al. reported ongoing decreases in MRSA and 
VRE rates after the de-escalation of contact precautions for MRSA and VRE infected or colonized patients with concurrent deployment of a robust, horizontal infection control platform, including hand hygiene, use of safety checklists, and chlorhexidine bathing [15••]. With this intervention, a $45 \%$ reduction in contact precaution burden was observed with an estimated $\$ 700,000$ dollars in cost saving from decreased gown and glove use. Similarly, Martin et al. reported ongoing reductions of MRSA and VRE infections after the deescalation of contact precautions in a major academic medical center with corresponding cost savings [16••]. More recently, Bearman and colleagues employed an interrupted time series analysis on the de-escalation of contact precautions and reported no significant (negative) impact on already decreasing 7-year trends of MRSA, VRE, and all HAIs [17••].

In a comprehensive literature review on the use of contact precautions for endemic pathogens, Morgan et al. summarized that no high-quality data supports or rejects the use of contact precautions for endemic MRSA or VRE prevention [18•]. At the time of publication, over 30 US hospitals did not employ contact precautions for the control of endemic MRSA or VRE. Until more definitive data are available, the authors proposed that the use of contact precautions for endemic MRSA/VRE in acute care hospitals should be guided by local needs and resources and not by mandate. This is underscored by a recent editorial urging hospitals to reconsider best use of contact precautions for endemic MRSA and VRE in the context of a broad approach to infection control which targets the highestvalue interventions [19॰]. Ongoing controversies in infection prevention raise questions about optimal strategies and expected patient safety outcomes.

\section{Surveillance Can Overdiagnose HAls: So What Is the Real HAI Incidence?}

Surveillance definitions by the CDC for HAIs favors sensitivity in diagnosis while minimizing subjectivity in case ascertainment. Estimates on the overdiagnosis of HAIs vary; however, a recent summary suggested that overdiagnosis of CAUTI is up to $37 \%$, CLABSI up to $30 \%$, C. difficile up to $53 \%$, and VAP up to $68 \%$ [20••]. Overdiagnosis of hospitalacquired infections through highly sensitive case definitions inflate the incidence of HAIs and calls into question the true incidence of harm. Further, this leads to strategies to minimize overtesting, which in turn results in a decrease in incident cases, giving the false impression of effective infection prevention. The HAI at the forefront of the charge for diagnostic stewardship is $C$. difficile. As laboratories nationwide adopted highly sensitive molecular testing platforms [21], hospitals saw their rates explode. Because inpatient colonization dramatically outnumbers true $C$. difficile infection incidence [22], many infection prevention programs realized that $C$. difficile rates would be directly proportional to testing volume. Testing volume remains the "missing denominator" In hospital comparisons of $C$. difficile HAIs, and thus, many center have spent the last few years devising ways to limit testing, hopefully while maintaining core infection prevention practices.

Like $C$. difficile, CAUTI rates have been found to be heavily influenced by the frequency of urine culturing, and NHSNdefined CAUTIs correlate poorly with clinically determined CAUTI events $[23 \cdot \bullet]$.

\section{The Diagnostic System Can Be Gamed: Infection Prevention Outcomes Are Misleading}

In an environment with comparative metrics, such as pay for performance, questionable practices emerge to improve infection prevention outcomes. Broadly, healthcare providers can conceal HAIs via a number of strategies and infection prevention personnel can also fail to report HAI cases [24]. A summary of practical interventions to game the system and get to zero was published by Horowitz [25••]. Interventions include screening urine on admission to ensure pyuria and discarding samples with less than 10 white blood cells/mm [3・•], to minimize the incidence of CAUTI. For CLABSI reduction, midline catheters could preferentially be used instead of PICC lines and only a single blood culture could be performed within $48 \mathrm{~h}$. With respect to surgical site infection incidence reduction, follow-up could be scheduled 30 days post-procedure and surgical teams could be coached to avoid the term purulent when describing a wound. These interventions would artificially decrease the incidence of healthcare-associated infections giving the misleading impression of getting to zero by true risk reduction practices. With $C$. difficile infection (CDI), hospitals can restrict testing and this might lead to underdiagnosis of true infections with resultant individual patient harm and risk for cross-transmission. Additionally, hospitals can embrace strategies wherein treatment for CDI is empiric (without testing), thus eliminating the potential for a HAI designation but leading to unnecessary antibiotic exposure [20••]. In terms of ventilator-associated events (VAE), these can be avoided by manipulating an individual patient's ventilator settings. Although doing so will lead to a decreased incidence of VAE, such strategies may directly harm patients [26]. Diagnostic or test stewardship purportedly attempts to reduce overdiagnosis and overtreatment of infection such as C. difficile and CAUTI by sending studies only on patients with specific clinical signs and symptoms of disease. Testing on the basis of a single non-specific symptom (e.g., fever) is discouraged [27•]. Test stewardship should benefit patients in less exposure to unnecessary testing and decreased antibiotic exposures. However, inadvertent harms from these practices have not yet been fully explored. Furthermore, the secondary 
gains in terms of reduction in reported HAI rates may blur the line between stewardship in testing and gaming the system. Healthcare centers may ultimately be pushed to pursue more and more restrictive ordering protocols in order to compete with peer institutions employing similar tactics.

\section{Human Beings as Chaotic Systems: the Cause and Effect Caveat}

Most natural systems play by the rules of chaos and are not linear [28]. Despite homeostasis, the human body is aperiodic and unpredictable in the long term. This is an important nuance as the human body is a chaotic, non-linear system. From this arises an important caveat. The simple cause and effect of an intervention preventing an outcome is not always reliable in the human body. Although medical treatments generally have predictable outcomes, medicine is unlike a physical science, one with better defined laws and outcomes. Infection prevention interventions are similarly effective like medical treatments and subject to variable outcomes.

\section{Hospital Senior Leadership: Safety Starts at the Very Top}

There is mounting evidence that leaders drive improvements in quality and safety. Hospital leaders create a sense of urgency, provide focus, create platforms for engagement, and advocate result-oriented approaches [29]. Particularly, in this age of Pay-For-Performance Systems, administrative perspective and engagement on management and change is critical [30॰]. Leaders must establish a sense of urgency, communicate the vision, empower the action, institutionalize change, and provide the necessary resources and holding both individuals and systems accountable. Without robust, engaged, and continued executive sponsorship, no infection prevention program will reach its stated goals. Maintaining engagement from hospital leadership is an ongoing challenge given competing administrative priorities.

\section{So How Much Can We Prevent HAls and How Hard Should We Try?}

The extent to which all HAIs can be prevented remains debatable and is subject to multiple considerations and barriers (Table 1). Eradication of infectious diseases is a lofty goal and has as of yet only been achieved for smallpox. As previously stated by Dellinger, the only foolproof way to ensure zero HAIs is not to admit patients to the hospital [31]. In 2003, following an overview of published reports, Harbarth and colleagues estimated that $10-70 \%$ of HAIs were preventable
[32]. A comprehensive estimate on the preventability of HAIs was subsequently published by Umscheid [33]. In a systematic review of interventions to reduce HAIs over last 10 years, with the current application of infection prevention science, it is believed that CLABSI may be reduced up to 65 $70 \%$, CAUTI up to $65-70 \%$, VAP up to $55 \%$, and SSI up to $55 \%$. A more recent systematic review and meta-analysis suggests that the sustained potential for HAI reduction ranges from 35 to $55 \%$ with current infection science [34••]. The authors argued that the preventable proportion of HAIs may reduce over time with improvements in safety resulting in a phenomenon of infection prevention diminishing returns [34••]. Thus, with the current infection prevention science, perhaps up to $70 \%$ of HAIs are potentially preventable when evidence based measures are effectively applied.

In 1978, Professor Herbert A. Simon of Carnegie Mellon University was awarded the Nobel Prize for his studies of decision-making in economics [35]. Professor Simon coined the term satisfice, a syncretic word derived from a combination of satisfy and suffice. He urged decision makers to satisfice either by finding optimum solutions for a simplified world or by finding satisfactory solutions for a more realistic world.

Under this paradigm, infection prevention programs should satisfice in the relentless pursuit of pragmatic and broadly applicable real-world solutions. In a viewpoint article, Edmond and Wenzel argue that horizontal infection prevention strategies should be the platform for all infection prevention programs [36]. Unlike vertical strategies that are characterized by interventions aimed at reducing risk from a single pathogen and commonly involve a microbiologic testing component, such as active detection and isolation of MRSA and VRE, horizontal infection prevention strategies focus on multipotent interventions aimed at reducing risk from all pathogens transmitted in the same mechanism-contact. Horizontal strategies include hand hygiene, chlorhexidine bathing, central line insertion bundle, ventilator bundle, and urinary catheter bundles.

Given the above, infection prevention may be conceptualized as outlined by Dellinger [37•]. An HAI is potentially preventable when all agreed upon evidence-based infection prevention interventions are administered with fidelity. An HAI is potentially unpreventable when an infection ensues despite application of every agreed upon measure for infection prevention. The primary focus is modifiable risk factors. For each HAI discovered, an investigation follows to define if every agreed upon measure for prevention was followed. If so, an apparently unpreventable HAI occurred; if not, there exists a critical and time-sensitive opportunity for HAI prevention improvement. Thus, we should relentlessly strive for zero potentially preventable infections as it is consistent with the Hippocratic Oath of primum non nocere, first do no harm. 
Table 1 Considerations and obstacles in striving for zero potentially preventable hospitalacquired infections

\begin{tabular}{|c|c|}
\hline Barrier & Comments \\
\hline $\begin{array}{l}\text { Avoid unrealistic expectations } \\
\text { in HAI prevention }\end{array}$ & $\begin{array}{l}\text { "Getting to zero" campaigns more consistent with } \\
\text { traditional quality improvement projects than } \\
\text { hospital epidemiology-based approaches. } \\
\text { Oversimplifies HAI reduction and leads to } \\
\text { unrealistic expectations. } \\
\text { Infection prevention science is inexact and } \\
\text { evolving. }\end{array}$ \\
\hline $\begin{array}{l}\text { Current status of implementing risk } \\
\text { reduction practices }\end{array}$ & $\begin{array}{l}\text { Evidence based infection prevention practices } \\
\text { are often incompletely implemented even in } \\
\text { high-quality clinical studies. }\end{array}$ \\
\hline Controversies in infection prevention & $\begin{array}{l}\text { The use of contact precautions for the control } \\
\text { of endemic MRSA and VRE is controversial } \\
\text { and of debatable benefit. } \\
\text { Optimal isolation strategies for endemic pathogens } \\
\text { are unknown. }\end{array}$ \\
\hline $\begin{array}{l}\text { Overdiagnosis with current surveillance } \\
\text { mechanisms }\end{array}$ & $\begin{array}{l}\text { Highly sensitive surveillance definitions label } \\
\text { infections in the absence of clinically } \\
\text { relevant disease. } \\
\text { Overdiagnosis may be driven by local clinician } \\
\text { practice as well as the laboratory procedural } \\
\text { differences. } \\
\text { Overdiagnosis calls into question the real } \\
\text { incidence of HAIs. }\end{array}$ \\
\hline The diagnostic system can be gamed & $\begin{array}{l}\text { Clinicians, infection prevention programs, and } \\
\text { hospitals can deploy techniques designed to } \\
\text { minimize HAI diagnoses. These may lead to } \\
\text { individual patient harm. } \\
\text { Perceived reductions in HAI incidence by gaming } \\
\text { the system leads to false conclusions about } \\
\text { preventability. }\end{array}$ \\
\hline Human beings as chaotic systems & $\begin{array}{l}\text { Medicine is not a physical science with precisely } \\
\text { defined laws and outcomes. } \\
\text { Infection prevention interventions, like medical } \\
\text { treatments, are subject to variable outcomes. }\end{array}$ \\
\hline Hospital leadership & $\begin{array}{l}\text { Without robust, engaged and continued executive } \\
\text { sponsorship, no infection prevention program } \\
\text { will reach its stated goals yet keeping leaders } \\
\text { engaged in safety is a challenge. }\end{array}$ \\
\hline
\end{tabular}

To maximize the application of horizontal infection prevention best practices, infection prevention personnel must consistently seek multimodal strategies. Risk reduction interventions must satisfice and be sustainable. Deployment of strategies must be evidence based, firmly grounded in the principles of implementation science and augmented by the application of a proven administrative framework, such as the Four Disciplines of Execution, which focuses on specific goal setting, regular process, and outcome audits along with individual and system accountability [38].

Further, epidemiologists must engage in an HAI prevention policy to define best prevention practices and specify key recommendations with tools to measure and improve compliance with safety processes [39]. There should be a demand for transparency of processes and outcomes so as to maximize both internal and public reporting of infection prevention performance. Additionally, critical next steps for infection prevention include an increased use of information technology to monitor and address HAI processes and outcomes and to nudge providers in all relevant infection practices. Strong economic analyses of infection prevention strategies are urgently needed to maximize cost/benefit guidance to help prioritize efforts and to minimize opportunity costs [40•]. Investments in the science of infection prevention should have a heavy focus on implementation science so as to effectively change theory into practice [41].

With a clear framework grounded in evidence-based strengths and limitations of current infection prevention science, pragmatism, cost/benefit-driven prioritization, and implementation science principles, infection prevention 
programs may clearly communicate expected processes and outcomes in the relentless quest for zero potentially preventable hospital-acquired infections.

\section{Conclusion}

Hospital-acquired infections result in significant morbidity, mortality and cost, obligating us to act. The concept of getting to zero HAIs is a soundbite as infection prevention science is inexact. Even high-quality studies have limitations and infection prevention processes are inconsistently implemented. Current infection prevention processes, such as contact precautions, can be controversial, raising questions about optimal strategies and expected outcomes. Diagnostic strategies and gaming can lead to inexact HAI incidence, false conclusions about preventability, and potential patient harm. Human beings are chaotic systems and do not always respond to linear mechanisms suggesting that not all interventions will have the desired result. With the current state of infection prevention science, perhaps up to $70 \%$ of HAIs are potentially preventable. Infection prevention personnel should relentlessly strive for zero potentially preventable HAIs as this is consistent with the mandate of "primum non nocere" (first do no harm) in the Hippocratic Oath. Health systems should seek practical solutions for real-world implementation of known risk reduction interventions. This includes the leveraging of information technology to assist with HAI prevention and surveillance and advocating for sound policies and improvements in infection prevention science. Hospital administration is key to achieving safety goals and must be engaged and leveraged at all time. Epidemiologists should strive for decisions based on cost/benefit and should be clear on expected HAI processes and outcomes. Infection prevention personnel and administrators should be aware of the potential for HAI "gaming" and not allow this to drive practices that could lead to patient harm. Zero potentially preventable HAIs requires a relentless pursuit of reliability and sustainability in the implementation of realworld infection prevention best practices.

Acknowledgements Authors would like to recognize Ms. Tina Olkonen for her assistance with preparing the final manuscript.

\section{Compliance with Ethical Standards}

Conflict of Interest Gonzalo Bearman, Michelle Doll, Kaila Cooper, and Michael Stevens declare that they have no conflict of interest.

Human and Animal Rights and Informed Consent This article does not contain any studies with human or animal subjects performed by any of the authors.

Publisher's Note Springer Nature remains neutral with regard to jurisdictional claims in published maps and institutional affiliations.

\section{References}

Papers of particular interest, published recently, have been highlighted as:

- Of importance

- Of major importance

1. Klevens RM, Edwards JR, Richards CL, et al. Estimating health care-associated infections and deaths in U.S. hospitals, 2002. Public Health Rep. 2007;122:160-6.

2. Zimlichman E, Henderson D, Tamir O, Franz C, Song P, Yamin $\mathrm{CK}$, et al. Health care-associated infections: a meta-analysis of costs and financial impact on the US health care system. JAMA Intern Med. 2013;173:2039-46.

3.• Magill SS, O'Leary E, Janelle SJ, et al. Changes in prevalence of health care-associated infections in US hospitals. N Engl Med. 2018;379:1732-44 Most up to date assessment of the burden of HAIs in the USA.

4.• Leapfrog Group. Healthcare associated infections. In: reports on hospital performance: 2018 report series. Available at: http://www. leapfroggroup.org/sites/default/files/Files/Leapfrog-Castlight $\%$ 202018\%20HAI\%20Report.pdf. Accessed November 1st 2018. Up to date assessment of HAI prevention performance.

5. Pronovost $\mathrm{P}$, Needham $\mathrm{D}$, Berenoltz $\mathrm{S}$, et al. An intervention to decrease catheter-related bloodstream infections in the ICU. N Engl J Med. 2006;355:2725-32.

6. Edmond MB. Getting to zero: is it safe? Infect Control Hosp Epidemiol. 2009;30:74-6.

7. Berenholtz SM, Pronovost PJ, Lipsett PA, Hobson D, Earsing K, Farley JE, et al. Eliminating catheter-related bloodstream infections in the intensive care unit. Crit Care Med. 2004;32:2014-20.

8. Timsit JF, Schwebel C, Geffroy A, et al. Chlorhexidineimpregnated sponges and less frequent dressing changes for prevention of catheter-related infections in critically ill adults: a randomized controlled trial. JAMA. 2009;25(301):1231-41.

9. Climo MW, Yokoe DS, Warren DK, Perl TM, Bolon M, Herwaldt LA, et al. Effect of daily chlorhexidine bathing on hospital-acquired infection. N Engl J Med. 2013;368:533-42.

10. Daneman N, Guttmann A, Wang X, Ma X, Gibson D, Stukel TA. The association of hospital prevention process and patient risk factors with the risk of clostridium difficile infection: a populationbased cohort study. BMJ Qual Saf. 2015;24:435-43.

11. Fakih MG, Heavens M, Ratcliffe CJ, Hendrich A. First step to reducing infection risk as a system: evaluation of infection prevention processes for 71 hospitals. Am J Infect Control. 2013;41:950-4.

12. Dhar S, Marchaim D, Tansek R, Chopra T, Yousuf A, Bhargava A, et al. Contact precautions: more is not necessarily better. Infect Control Hosp Epidemiol. 2014;35:213-21.

13. Bearman G, Stevens MP. Control of drug-resistant pathogens in endemic settings: contact precautions, controversies and a proposal for a less restrictive alternative. Curr Infect Dis Rep. 2012;14:620-6.

14. Harris AD, Pineles L, Belton B, Johnson JK, Shardell M, Loeb M, et al. Universal glove and gown use and acquisition of antibiotic-resistant bacteria in the ICU: a randomized trial. JAMA. 2013;310:1571-80.

15.• Edmond MB, Bearman G, Masroor N, Steven M, Ober J. The impact of discontinuing contact precautions for VRE and MRSA on device-associated infections. Infect Control Hosp Epidemiol. 2015;36:978-80 Important publication on the controversy of discontinuing contact precautions for endemic pathogens: MRSA and VRE.

16.• Martin EM, Russell D, Rubin Z, Humphries R, Grogan TR, Elashoff D, et al. Elimination of routine contact precautions for endemic methicillin-resistant Staphylococcus aureus and vancomycin-resistant Enterococcus: a retrospective quasi- 
experimental study. Infect Control Hosp Epidemiol. 2016;37: 1323-30 Important publication on the controversy of discontinuing contact precautions for endemic pathogens: MRSA and VRE.

17.• Bearman G, Abbas S, Masroor N, et al. Impact of discontinuing contact precautions for methicillin-resistant Staphylococcus aureus and vancomycin-resistant Enterococcus: an interrupted time series analysis. Infect Control Hosp Epidemiol. 2018;39:676-82 First interrupted time series analysis published on discontinuing contact precautions for endemic pathogens: MRSA and VRE.

18. Morgan DJ, Bearman G, Murthy R, et al. Reconsidering contact precautions for endemic methicillin-resistant Staphylococcus aureus and vancomycin-resistant Enterococcus. Infect Control Hosp Epidemiol. 2015;36:1163-72 Important summary of contact precautions controversy and evidence.

19. Morgan DJ, Wenzel RP, Bearman G. Contact precautions for endemic MRSA and VRE time to retire legal mandates. JAMA. 2017;318:329-30 Editorial advocating the retirement of legal mandates for MRSA and VRE control.

20.• Madden GR, Weinstein RA, Sifri CD. Diagnostic stewardship for healthcare-associated infections: opportunities and challenges to safely reduce test use. Infect Control Hosp Epidemiol. 2018;39: 214-8 Important paper that highlights the evolving role of diagnostic stewardship in infection prevention.

21. Burnham CA, Carroll KC. Diagnosis of clostridium difficile infection: an ongoing conundrum for clinicians and clinical laboratories. Clin Microbiol Rev. 2013;26:604-30.

22. Morgan DJ, Leekha S, Croft L, et al. The importance of colonization with clostridium difficile on infection and transmission. Curr Infect Dis Rep. 2015;17:499.

23.•- Leekha S, Preas MA, Hebden J. Association of national healthcare safety network-defined catheter-associated urinary tract infections with alternate sources of fever. Infect Control Hosp Epidemiol. 2015;36:1236-8 Paper highlights the potential inaccuracies of national surveillance definitions.

24. Ider BE, Adams J, Morton A, Whitby M, Clements A. Gaming in infection control: a qualitative study exploring the perceptions and experiences of health professionals in Mongolia. Am J Infect Control. 2011;39:587-94.

25.• Horowitz HW. Infection control II: a practical guide to getting to zero. Am J Infect Control. 2016;44:1075-7 Important editorial highlighting potential mechanisms to game HAI surveillance.

26. Klompas M, Berra L. Should ventilator-associated events become a quality indicator for ICUs? Respir Care. 2016;61:723-36.

27. Mullin KM, Kovacs KS, Fatica C, et al. A multifaceted approach to reduction of catheter-associated urinary tract infections in the intensive care unit with an emphasis on "stewardship of culturing". Infect Control Hosp Epidemiol. 2017;38:186-8 Paper highlights diagnostic stewardship to reduce catheter associated urinary tract infections.

28. Ives, Crystal, Human beings as chaotic systems. http://www.fractal. org/Life-Science-Technology/Publications/Human-beings-asfractal-systems.pdf. Accessed 10.23.18.

29. Bowen DJ. Leading quality and safety. Getting to zero harm must start at the top. Healthcare Executive. 2015;30:8.

30. Vokes RA, Bazzoli GJ, Bearman G. Hospital-acquired infections under pay-for-performance systems: as administrative perspective on management and change. Curr Infect Dis Rep. 2018;20:35 Editorial underscores the importance of executive leadership to reach safety goals.

31. Dellinger EP. Prevention of hospital-acquired infections. Surg Infect. 2016;17:422-6.

32. Harbarth S, Sax H, Gastmeier P. The preventable proportion of nosocomial infections: an overview of published reports. J Hosp Infect. 2003;54:258-66258-66.

33. Umscheid CA, Mitchell MD, Doshi JA, Agarqal R, Williams K, Brennan PJ. Estimating the proportion of healthcare-associated infections that are reasonably preventable and the related mortality and costs. Infect Control Hosp Epidemiol. 2011;32:101-14.

34.• Schreiber PW, Sax H, Wolfensberger A, Clack L, Kuster SP, Swissnoso. The preventable proportion of healthcare-associated infections 2005-2016: systematic review and meta-analysis. Infect Control Hosp Epidemiol. 2018;39:1277-95 Most up-todate assessment of proportion of preventable healthcareassociated infections.

35. The Sveriges Riksbank prize in economic sciences in memory of Alfred Nobel 1978. NobelPrize.org. https://www.nobelprize.org/ prizes/economics/1978/press-release/. Accessed October 27, 2018.

36. Wenzel RP, Edmond MB. Infection control: the case for horizontal rather than vertical interventional programs. Int J Infect Dis. 2010;14:S3-5.

37. Dellinger EP. Prevention of hospital-acquired infections. Surg Infect. 2016:17422-6 Publication defines and distinguishes potentially preventable vs apparently unpreventable HAIs.

38. McChesney C, Covey S, Huling J. The 4 disciplines of execution: achieving your wildly important goals. New York: Free Press; 2012.

39. Richards C. Getting to zero: an emergency policy framework for the elimination of hospital-associated infections. Infect Control Hosp Epidemiol. 2009;30:71-3.

40. Gray J. Infection control: beyond the horizon. J Hosp Infect. 2015;89:237-40 Publication highlights critical next steps in infection prevention science.

41. Kahn KL, Mendel P, Baker DP. Lessons learned and future directions: the national response for preventing health-care associated infections. Med Care. 2014;52:385. 